\title{
Abstracts for the 15th World Congress for Disaster and Emergency Medicine
}

\author{
13-16 May 2007 \\ Amsterdam, The Netherlands
}

\footnotetext{
We regret to inform the readers that some errors were identified in some of the abstracts from the 15 th World Congress for Disaster and Emergency Medicine in a recently printed issue of Prehospital and Disaster Medicine. The corrected abstracts are printed here.
}

\author{
Poster Presentation: Nursing and Paramedic Issues \\ Evaluation of Inspiratory Impedance Valve (ITD) in EMS Cardiac \\ Arrest Victims \\ P. Padjen; D. Martin-Lightfoot \\ University of Wisconsin Hospital and Clinics, Madison, Wisconsin USA
}

Introduction: The effectiveness of cardiopulmonary resuscitation (CPR) is dependent on the technique of the rescuers and the time after arrest that CPR is initiated to the victim. Incomplete chest wall recoil during the decompression phase of CPR increases intra-thoracic pressure and decreases coronary and cerebral perfusion. The Inspiratory Impedance Valve (ITD) prevents unnecessary air from entering the lungs during the decompression phase.

Objective: The purpose of this study was to clinically evaluate the ITD.

Research Questions:

1. Was there a statistically significant difference in the relationship between cardiac arrest survival rates of cardiac arrest victims receiving manual CPR with intubation or bag-valve-mask airway support and cardiac arrest victims receiving manual CPR with intubation or bagvalve-mask airway support with an ITD?

2. What was the perception of paramedic satisfaction after use of the ITD? Methods: Evaluation methodology was used to clinically evaluate the ITD, and qualitative methodology was used to assess the paramedics' impressions of the product. The study consisted of witnessed or un-witnessed arrests and CPR was initiated within minutes. Rhythms included PEA, asystole, pulseless VT, and VF. The patients included 39 ITD patients and 36 non-ITD patients. The participants all were firefighter EMT-B or EMT-P who were ITD trained.

Results: The code was discontinued in the field for patients with and without ITD (ITD chi-square $\left(\chi^{2}\right) 11.189, p=0.001$ ). Return of spontaneous circulation upon arrival to the emergency department was $\chi^{2} 3.654, p=0.56$. A total of $96 \%$ of paramedics were satisfied with the ITD. Two patients survived, but 16 eventually died in the emergency department.

Limitations: Some data fields were not completed.

Conclusions: The results of this study are in alignment with AHA support of the ITD.

Keywords: cardiac arrest; cardiopulmonary resuscitation; emergency medical services; inspiratory impedance valve

Prebosp Disast Med 2007;22(3):256

\section{Oral Presentation: Preparedness Ontario CritiCall Program and Provincial Disaster Management K. Bachynski \\ Ontario CritiCail Program, Hamilton, Ontario Canada}

Since 1996, the Ontario CritiCall Program has been Ontario's sole emergency patient referral program. CritiCall ("medical 9-1-1" for physicians) is a one-number-to-call, 24-hour-a-day, physician referral based service providing care across the province of Ontario, Canada. Geographically, Ontario is comparable in size to the combined size of Spain and France. The approach of this 
presentation is patient and physician-to-physician focused, which allows for immediate medical management and determination of appropriate care. A key resource is the Internetbased Canadian Provincial Bed and Resource Registry.

This computer registry provides information regarding the physician on-call, pager number, and resource availability by specialty, including all beds at local, regional, or provincial level hospitals. All information within the resource registry is reportable, providing an extensive, provincial, resource database in real time along with a provincially statistical patient database. During the 2003 Severe Acute Respiratory Syndrome (SARS) outbreak, all patients were transferred via CritiCall, which maintained epidemiological links, effectively used limited specialized resources, and provided significant statistical tracking on all of the above information.

CritiCall can be used in a disaster situation using the same methodology. Disaster "fan-out" alerts are sent to many agencies both manually and electronically. Alerts are for local, regional, and/or provincial hospitals, emergency medical services, and the Ministry of Health to confirm and create bed availability, alert all specialists, and control non-disaster'related patient flow. During all disaster events, such as the 2002 ice storm and the 2003 SARS outbreak, the appropriate and methodical transfer of patients is paramount and statistical tracking is invaluable.

Keywords: Canada; Criticall; databases; patient referrals; severe acute respiratory syndrome

Prehosp Disast Med 2007;22(3):258-259

\section{Oral Presentation: Preparedness}

Improving Hospital Preparedness for Radiological Terrorism: Perspectives from Emergency Department Physicians and Nurses

\section{S.M. Becker}

University of Alabama-Birmingham, Birmingham, Alabama USA

In any large-scale terrorist event involving radioactive materials, hospital emergency departments will play a pivotal role in providing care. In light of this, numerous efforts currently are in process to improve hospital preparedness. However, for such initiatives to be effective, there must be an understanding of the practical concerns, needs, and views of front-line hospital staff. This presentation will report findings from study of emergency department physicians' and nurses' perspectives on radiological terrorism issues. It was funded by the United States Centers for Disease Control and Prevention and performed in 2005 and 2006 in the Northeastern, Southern, and Western regions of the US involving a series of 10 focus groups. The issues examined were the: (1) the principal preparedness challenges for hospitals, emergency departments, and staff; (2) critical information needs and preferred information sources; (3) views of current training and future training needs; (4) views of current response plans and protocols; and (5) perspectives on potential difficulties and impediments that could affect readiness. Key findings from the study will be reviewed in this presentation, and the implications for hospital preparedness and response will be explored. Keywords: hospitals; nurses; physicians; preparedness; radiological terrorism

Prebosp Disast Med 2007:22(3):259

\section{Oral Presentation: Preparedness}

Benchmarking Hospitals for Hurricane Evacuation

L. Downey; ${ }^{1}$ W.K. Andress, ${ }^{2}$ C.H. Schultz

1. Tulane School of Public Health, Baton Rouge, Louisiana USA

2. Christus Health Systems, USA

3. Emergency Medical Services and Disaster Medical Sciences Fellowship, University of California-Irvine School of Medicine, Orange, California USA

The 2005 hurricane season on the Gulf Coast of the United States underscored how quickly hospitals can shift from being care providers during a disaster to being victims in need of assistance themselves. Four weeks after Hurricane Katrina, seven system hospitals in southeast Louisiana and southwest Texas that were serving the population surge of the New Orleans patients and evacuees, were either partially or fully evacuated as a result of Hurricane Rita. This study builds on the Northridge hospital benchmarking tool for evacuations during an earthquake (Schultz et al 2005), and enhances its design for benchmarking hospitals for hurricane evacuation. Study areas include: (1) hospital demographics; (2) disaster plan characteristics; (3) planning lessons for individual hospitals; (4) hospital decision making and incident command; (5) movement of patients within the facility; and (6) movement of patients to other facilities. Lessons learned from multiple communities across the region will be discussed in the results, and key planning areas that strengthen hospital evacuation planning will be identified.

Keywords: benchmarking; evacuation; hospital; Hurricane Katrina Hurricane Rita;

Prebosp Disast Med 2007;22(3):259

\section{Oral Presentation: Life-Tbreatening Situations in Daily Emergencies and Disasters \\ Emergency Medical Evacuation of Patients with Severe Lung Failure using Miniaturized Extracorporeal Assistance Devices}

M. Arlt; A. Philipp; M. Zimmermann; K. Taeger;

F.X. Schmid

University Hospital Regensburg, Regensburg, Germany

Objective: An experience with two new miniaturized extracorporeal assistance devices (EADs) for emergency, interhospital transfer for adult patients experiencing severe lung failure was analyzed. The utilization, efficiency, and safety of the new assistance devices were characterized. Patient preparation, including cannulation were described, and the care and precautions en route were reported.

Methods: Between March 2001 and February 2007,EADs were used to facilitate medical emergency evacuation for 19 adult patients with severe hypoxemic/hypercarbic respiratory failure $(n=15)$, or combined respiratory and cardiac failure $(n=4)$. Extracorporeal assistance devices were used to access the percutaneous vessels. The technique included pumpless extracorporeal lung assist (PECLA) in 15 patients. Closed-loop, extracorporeal circulation with a centrifugal pump unit for arterio-venous life support $(n=3)$ and veno-venous extracorporeal membrane oxygenation $(n=1)$ was assessed with the new Emergency Life Support System (ELS). The ELS and PECLA systems are small enough to be placed on a standard gurney. 
Results: None of the patients died en route. Air medical evacuation was used for 13 patients, and six patients were transported via ground ambulance. Survival was $40 \%$ in the PECLA group and $75 \%$ for the ELS patients. During extracorporeal assistance, no technical complication occurred. The patient-related complication was two cases of limb ischemia due to the arterial cannula.
Conclusions: The application of miniaturized extracorporeal assistance devices enables the secure transportation of critically ill patients without technical or personnel support. Oxygen delivery can be restored rapidly and bloodflow can be ensured en route.

Keywords: emergency medical services; evacuation; extracorporeal assistance devices; lung failure; transport Prehosp Disast Med 2007;22(3):259-260 